\title{
Evaluation of role of mean corpuscular volume and RBC-histogram in analysing anaemias as a rapid method in comparison to peripheral smear evaluation
}

\author{
O. Sirisha ${ }^{1}$, M.N.P. Charan Paul ${ }^{2}$, P. Yujwal Raj ${ }^{3}$ \\ ${ }^{1}$ Dr. O. Sirisha, Assistant Professor, ${ }^{2}$ Dr. M.N.P. Charan Paul, Associate Professor; ${ }^{1,2}$ authors are affiliated with \\ Department of Pathology, Bhaskar Medical College, Moinabad, Telangana, ${ }^{3}$ Dr. P. Yujwal Raj, Consultant, Public \\ Health Expert, Telangana, Andra Pradesh, India.
}

Corresponding Author: Dr. O. Sirisha, Department of Pathology, Bhaskar Medical College, Moinabad, Telangana, Andhra Pradesh, India. E-mail id: psirisharaj@gmail.co

\begin{abstract}
Objective: From ages, Peripheral smear evaluation is being considered as the gold standard for evaluation of anaemias. Various other parameters have been assessed for their role as a substitute for peripheral smear. The present study evaluates Mean Corpuscular Volume (hereby referred to as MCV) and RBC-histograms if they are reliable as substitutes for the smear evaluation. Materials \& Methods: This is a prospective study conducted at the department of Clinical Pathology, Bhaskar Medical College. A total of 129 cases diagnosed as having anaemia were included in the study. Initially each case was analysed based upon the cell counter values, RBC indices \& RBC histogram. A detailed peripheral smear evaluation was done later for each case. The results were compared and analysed statistically. Sensitivity, Specificity and Positive, Negative predictive values were calculated. Results: Female cases outnumbered the male patients. Microcytic hypochromic anaemia was the most common type (61\%); MCV was able to detect $75 \%$ of cases of macrocytic anaemia and $76 \%$ of cases of microcytic hypochromic anaemia. In contrast, $65 \%$ of cases of dimorphicanaemia had MCV values in the normal range. RBC-histograms were able to detect $63 \%$ of cases, $83 \%$ of cases with macrocytic anaemia, only $41 \%$ of patients had the characteristic double-peak on histograms. Conclusion: Though MCV and RBC histograms can detect most of the cases of macrocytic anaemia and microcytic anaemias with accuracy, a case with normal MCV and increased RDW requires a peripheral smear examination as the findings were variable in dimorphic anaemia.
\end{abstract}

Key words: Mean Corpuscular Volume, Cell counter, Histogram, Sensitivity.

\section{Introduction}

Anaemia is a significant problem in developing countries. Anaemia is the most prevalent nutritional deficiency disorder in the world [1]. It affects all age groups but the most vulnerable are preschool-age children, pregnant women, and non-pregnant women of childbearing age. Globally, anaemia affects 1.62 billion people, which corresponds to $24.8 \%$ of the population [2].The highest prevalence of anaemia exists in the developing world where its causes are multi-factorial.

National Family Health Survey statistics reveal that every second Indian woman is anaemic and one in every five maternal deaths is directly due to anaemia [3]. In the age where a short turnover time for generation of reports is one of the criteria for quality of

Manuscript received: $2^{\text {nd }}$ February 2019

Reviewed: $10^{\text {th }}$ February 2019

Author Corrected: $15^{\text {th }}$ February 2019

Accepted for Publication: $19^{\text {th }}$ February 2019 the diagnostic labs, cell counters have become indispensable in evaluation of complete blood picture. Finally, in case of equivocal results, pathologists turn to peripheral smear for final opinion. In a laboratory where there are large number of cases per day for evaluation, it becomes mandatory to assess most of the cases based on automation. The search for the best automation is still ongoing, many parameters obtained on automated cell counters are screened for accuracy in comparison to the peripheral smear findings.

The present study is conducted in a tertiary care centre and the patient population is a rural population where awareness of anaemia and its consequences are largely neglected. The study aimed to compare the findings of $\mathrm{MCV}$ and RBC-histogramin relation to peripheral smear findings. The objective was to analyse if both the parameters would be useful in screening large rural 
patient populations for anaemia and categorise them with nearest accuracy. If possible, to do so, the parameters can be used to screen and categorise

\section{Materials \& Methodology}

Study setting: The present study was conducted at the Department of Clinical Pathology, Bhaskar Medical College, Moinabad. A totalof 129 cases were included in the study.

Sampling method: All the patients presenting with symptomatic anaemia or diagnosed for the first time with anaemia based on haematological findings who presented to the General Medicine Outpatient department were included in the study.

Inclusion criteria: Patients aged between 1 and 70 years of age diagnosed for the first time with anaemia were included in the study.

Exclusion criteria: Known cases of anaemia receiving treatment, pregnant women, age less than 1 year, anaemia due to defined causes like thyroid disorders, malignancies and other disorders were all excluded from the study as the treatment received by them may alter the results.

Sample collection: The clinical details, signs and symptoms of the patients, the cell counter findings and peripheral smear findings were recorded in a detailed protocol. The reference standards for all the parameters were clearly defined based on Indian reference standards.

Apparatus required: The cell counter used was that of SYSMEX -XP series 3-partcounter. Peripheral smear was stained with Leishman's stain and evaluated. Whole blood samples collected in EDTA vacutainers were used for sample collection.

Permissions: Institutional Ethics committee permission was taken before the start of the study. The study required no surgical procedure and hence no special consent for any procedure was taken from the patients.

Reference ranges: Standard reference ranges from the National Health Portal of India; ACCC press.

\section{Haemoglobinlevels [4]:}

Men: $13.8-17.2 \mathrm{gm} / \mathrm{dl}$

Women: $12.1-15.1 \mathrm{gm} / \mathrm{dl}$

Children: $11-16 \mathrm{gm} / \mathrm{dl}$

MCV[4]: Normal value MCV.

\begin{tabular}{|c|c|c|}
\hline & Male & Female \\
\hline 2-6 years & $76.8-83.3$ & $77.7-84.1$ \\
\hline 6-12 years & $78.2-83.9$ & $79.5-85.2$ \\
\hline 12-18 years & $80.8-86.6$ & $82.1-87.7$ \\
\hline$>18$ years & $81.2-94.0$ & $78.5-96.4$ \\
\hline
\end{tabular}

Classification of severity of anaemia [5]:

\begin{tabular}{|c|c|c|c|}
\hline & Mild & Moderate & Severe \\
\hline 6 months -5 years & $10-10.9$ & $7-9.9$ & $<7$ \\
\hline $5-11$ years & $11-11.4$ & $8-10.9$ & $<8$ \\
\hline $12-14$ years & $11-11.9$ & $8-10.9$ & $<8$ \\
\hline Non-pregnant women & $11-11.9$ & $8-10.9$ & $<8$ \\
\hline Men & $11-12.9$ & $8-10.9$ & $<$ \\
\hline
\end{tabular}


Original Research Article

The peripheral smear findings were taken as Gold standard and we compared MCV as well as RBC histograms with the smear findings. The sensitivity, specificity, positive predictive value (PPV) and negative predictive value (NPV) were calculated using the $2 \times 2$ square method and the results analysed.

Histogram interpretation: If the graph starts before $34 \mathrm{fl}$ and touches the baseline before $150 \mathrm{fl}$, they are considered to be a left shift indicates micro erythrocytosis. If the graph starts after $34 \mathrm{fl}$ and touches the base line after 150fl, they are considered to be a right shift indicates macro erythrocytosis. If graph starting at 34fl and ending between 225-250 FL, they are a broad base. If RBCs Populations has two morphologies, then the graph will have two peaks representing to their respective morphology and called as dimorphic red cells. If the RBCs population will have single lineage of cells seen, the graph will be constraint and will look like short peak [6].

\section{Results}

A total of 129 patients were assessed with a peripheral smear, RBC-histogram and MCV values. The Male: Female ratio was 1: 2. Thirty-four (34\%) of the female patients were in the age group of $20-40$ years. $45 \%$ of male patients were below the age of 20 years (Table 1). On classifying anaemia according to severity, $59 \%$ of patients had moderate anaemia; $34 \%$ were classified as having severe anaemia (Table 2). When anaemias were classified based on peripheral smear findings, $61 \%$ were microcytic hypochromic anaemia, $20 \%$ were normocytic normochromic anaemia and $13 \%$ were classified as dimorphic anaemia (Table 3).

Tables-1: Age and sex distribution $(n=129)$

\begin{tabular}{|c|c|c|}
\hline Age & Male & Female \\
\hline $1-10$ years & 9 & 4 \\
\hline $11=20$ years & 12 & 17 \\
\hline $21-30$ years & 2 & 26 \\
\hline $31-40$ years & 6 & 18 \\
\hline $41-50$ years & 6 & 9 \\
\hline $51-60$ years & 2 & 5 \\
\hline $61-70$ years & 5 & 3 \\
\hline $71-80$ years & 4 & 1 \\
\hline
\end{tabular}

Table-2: Classification of anaemia as per severity $(n=129)$

\begin{tabular}{|c|c|}
\hline Severity & Number of patients \\
\hline Mild & 8 \\
\hline Moderate & 76 \\
\hline Severe & 45 \\
\hline
\end{tabular}

Table-3: Morphological classification of anaemia $(n=129)$

\begin{tabular}{|l|c|}
\hline Type of anaemia & Number of patients \\
\hline Normocytic/Normochromic & 26 \\
\hline Microcytic/Hypochromic & 78 \\
\hline Macrocytic anaemia & 8 \\
\hline Dimorphic anaemia & 17 \\
\hline
\end{tabular}

When MCV was compared with peripheral smear findings (Table 4), $70 \%$ of cases with normocytic normochromic anaemia had MCV within the normal range. $75 \%$ of macrocytic anaemia cases had MCV greater than the normal range; $76 \%$ of microcytic hypochromic anaemia cases had MCV values lesser than the normal range. In contrast $65 \%$ of cases of dimorphic anaemia had MCV values within the normal range. When the RBC histograms were compared with the peripheral smear findings (Table 5), 75\% of cases with normocytic normochromic anaemia had normal histogram; $63 \%$ of cases with microcytic hypochromic anaemia had left shift in the histogram; $83 \%$ of cases with macrocytic anaemia had right shift in the histogram. In contrast only $41 \%$ of the patients with dimorphic anaemia had the characteristic "doublepeak" on histogram and $53 \%$ of the patients with dimorphic anaemia had either a right shift or a left shift of the histogram depending upon the predominant population of red cells. 
Table-4: Comparison of MCV with peripheral smear $(n=129)$.

Original Research Article

\begin{tabular}{|c|c|c|c|c|}
\hline MCV & $\begin{array}{c}\text { Normocytic/ } \\
\text { Normochromic }\end{array}$ & Macrocytic & $\begin{array}{c}\text { Microcytic/ } \\
\text { hypochromic }\end{array}$ & Dimorphic anaemia \\
\hline Normal & 14 & 2 & 19 & 11 \\
\hline Increased & 3 & 6 & 1 & 4 \\
\hline Decreased & 3 & 0 & 64 & 2 \\
\hline
\end{tabular}

Table-5: Comparison of RBC-histogram with peripheral smear $(n=129)$

\begin{tabular}{|l|c|c|c|c|}
\hline RBC-histogram & Normocytic/Normochromic & Microcytic & Macrocytic & Dimorphic anaemia \\
\hline Normal & 21 & 29 & 1 & 3 \\
\hline Left shift & 3 & 49 & 0 & 4 \\
\hline Right shift & 4 & 0 & 7 & 1 \\
\hline Double peak & 0 & 0 & 0 & 7 \\
\hline
\end{tabular}

When RBC-histogram was analysed for evaluation of anaemias in comparison to peripheral smear as GOLD STANDARD, for normocytic anaemia, an NPV of 90\% was obtained; in microcytic anaemia, PPV was $88 \%$; in macrocytic anaemia, specificity was $96 \%$ and NPV was $99 \%$. In case of dimorphic anaemia, specificity and PPV were $100 \%$ and NPV was $96 \%$.

Table-6: Sensitivity, Specificity, Positive \& Negative predictive values of MCV $(n=129)$.

\begin{tabular}{|l|c|c|c|c|}
\hline & Sensitivity & Specificity & PPV & NPV \\
\hline Normocytic/Normochromic & 70 & 70 & 30 & 92 \\
\hline Microcytic/hypochromic & 76 & 88 & 93 & 67 \\
\hline Macrocytic & 75 & 93 & 43 & 98 \\
\hline Dimorphic anaemia & 35 & 68 & 07 & 76 \\
\hline
\end{tabular}

Table-7: Sensitivity, Specificity, Positive \& Negative predictive values of RBC-histogram (n= 129)

\begin{tabular}{|l|c|c|c|c|}
\hline & Sensitivity & Specificity & PPV & NPV \\
\hline Normocytic/Normochromic & 75 & 65 & 38 & 90 \\
\hline Microcytic/hypochromic & 63 & 86 & 88 & 60 \\
\hline Macrocytic & 83 & 96 & 50 & 99 \\
\hline Dimorphic anaemia & 41 & 100 & 100 & 92 \\
\hline
\end{tabular}

\section{Discussion}

Women's health in India is facing a serious nutritional challenge, with the country on the one hand grappling with the largest number of anaemic women in the world[7]. Anaemia is a major killer in India. Statistics reveal that every second Indian woman is anaemic and one in every five maternal deaths is directly due to anaemia [8].

Anaemia spares none; it affects both adults and children of both sexes, although pregnant women and adolescent girls are most susceptible and most affected by this disease. The results on the prevalence and deaths due to anaemia are still staggeringly high despite the government having initiated many health programmes and allocated large amounts of finances over the last three decades to combat this disease[9]. In summary, the current situation is:

- One in every two Indian women (56\%) suffers from some form of anaemia

- 4 out of every 5 children in the age of 6-35 months suffer from anaemia [10].

- $20 \%$ of the maternal deaths are due to anaemia and anaemia indirectly contributes to another $40 \%$ of maternal deaths

- Maternal mortality staggeringly high at 454 per every 100,000 live births [11]. 
Original Research Article

The present study was conducted at the department of Clinical Pathology, Bhaskar Medical College. A total of 129 cases were diagnosed as having anaemia. Out of them, 46 were males, 83 were female patients. In the study by Sandhya et al, $53 \%$ of patients were females [12]. In the study by Sundara Rao et al, $70 \%$ of patients were females [13]. In the study by Kushwaha et al, 40\% of patients were females [14].

In the present study, $34 \%$ of female patients were in the age group of $20-40$ years, $45 \%$ of male patients were below theage of 20 years. In the study by Sundararao et al, maximum cases were found in the 30-40-year age group. In the study by Sandya et al, maximum number of cases were in the age group of $30-40$ years.

The present study clearly indicated that the factors responsible for anaemia in males and females were different. Menstruation, pregnancy and nutritional status are the main factors affecting haemoglobin levels in women while nutritional status is playing an important role in males. In the present study, anaemia was classified based on the severity - 59\% of cases had moderate anaemia; $34 \%$ of cases had severe anaemia.

The above finding indicated that most of the patients do not present clinically until haemoglobin levels are reduced well below the normal range. This necessitates frequent testing of higher risk groups like females beyond adolescence; those with a family history of anaemia and people with malnutrition.

When anaemias were classified according to the peripheral smear findings, the commonest type was microcytic hypochromic anaemia (61\%) followed by normocytic normochromic anaemia (20\%) and dimorphic anaemia (13\%). The observation indicated that iron deficiency was far more common than folate and B12 deficiency in the study population. In the study by Sandya et al, $61 \%$ cases were microcytic hypochromic anaemia; $17 \%$ were normocytic anaemia. In the study by Singla et al, $76 \%$ of cases were diagnosed as microcytic hypochromic anaemia [15]. In the study by Jain et al, $40 \%$ of cases were microcytic hypochromic anaemia [16].

The present study considered Peripheral smear opinion as the Gold Standard for the evaluation of anaemia. When MCV was compared with the peripheral smear findings, $70 \%$ of cases of normocytic normochromic anaemia had normal values; $75 \%$ of cases with macrocytic anaemia had increased values; $76 \%$ of cases of microcytic hypochromic anaemia had lesser values. In case of dimorphic anaemia, $65 \%$ of cases had normal MCV-hence would have been diagnosed as normocytic anaemia if peripheral smear was not evaluated in these cases. RDW plays an important role in these cases - if it is high, then a further assessment is necessitated. Thepresent study also compared RBC- histogram findings with peripheral smear findings - in $75 \%$ of cases with normocytic anaemia the RBC- histogram was a normal curve; in microcytic anaemia, $63 \%$ of cases had a left shift; in macrocytic anaemia $83 \%$ of cases had a left shift.

In case of dimorphic anaemia only $41 \%$ of cases had the classic "double peak" and approximately $30 \%$ of the cases had a normal curve on histogram. Before the smear was evaluated in cases of dimorphic anaemia, RDW was an important clue.

If it was elevated, even if $\mathrm{MCV}$ and $\mathrm{RBC}$-histogram were normal, the smear showed two or more populations of cells. However, dimorphic anaemia is a broad entity and hence a smear was always mandatory to assess the abnormal cells. The reason for the cases of dimorphic anaemia to show a normal histogram curve is that histogram has a very broad range - recognises cells between $34 \mathrm{fl}$ and $250 \mathrm{fl}$ as race; though the MCV has a shorter range, it was also not identifying all cases of dimorphic anaemia as MCV is an average value and hence is very susceptible to smallchanges in the MCV values.

In the study by Sandyaetal, out of the $17 \%$ of normocytic normochromic anaemia $8 \%$ showed normal histogram and $9 \%$ showed mild broad base curve histogram. Out of the $61 \%$ of microcytic hypochromic anaemia, 4\% were normal histogram, 27\% were left shift histogram, 26\% were broad base curve histogram, $2 \%$ short peak histogram and $2 \%$ abnormal (bimodal) histogram.In the study by Singla et al, out of $43(19.54 \%)$ cases of normocytic normochromic anaemia, 26(11.8\%) showed normal curve and $17(7.72) \%$ showed broad base curve.

Out of $140(63.23 \%)$ cases of microcytic hypochromic anaemia $7(3.18 \%)$ were normal, $60(27.27 \%)$ showed left shift curve, 60 (27.27\%) showed broad base curve, $7(3.18 \%)$ showed bimodal curve histogram. and 6 $(2.72 \%)$ showed short peak. Out of total $28(12.7 \%)$ cases of dimorphic anaemia, 9(4)\% showed normal curve, $4(1.81 \%)$ showed left curve, right curve $9(4 \%)$, broad base curve $4(1.81 \%)$ and $2(0.9 \%)$ showed bimodal curve.

The present study also calculated the sensitivity, specificity, positive and negative predictive values of $\mathrm{MCV}$ and RBC-histograms in assessing the various anaemias (Table 6 \& 7). When normocytic anaemia was 
assessed, the sensitivity \& specificity of MCV were low $(70 \%)$ but the negative predictive value was high (92\%); in microcytic anaemias, the PPV was 93\%; in Macrocytic anaemias, specificity was $93 \%$ and in dimorphic anaemia, all the predictive parameters were significantly low. When RBC-histogram was analysed for evaluation of anaemias in comparison to peripheral smear as Gold Standard, for normocytic anaemia, an NPV of $90 \%$ was obtained; in microcytic anaemia, PPV was $88 \%$; in macrocytic anaemia, specificity was $96 \%$ and NPV was $99 \%$.

In case of dimorphic anaemia, specificity and PPV were $100 \%$ and NPV was $96 \%$. The present study was the only one of this type which calculated the role of predictive parameters in analysing the role of MCV and RBC-histograms in analysing anaemias before the evaluation of peripheral smear.

\section{Conclusion}

MCV and RBC-histograms impart very valuable and importantly very early and rapid information about the $\mathrm{RBC}$ status of the patient in anaemias. But the sensitivity and specificity of MCV being low in all types of anaemias, it's use as a screening test is of doubtful value.

However, the positive predictive values being high for microcytic anaemia, negative predictive value being high for macrocytic anaemia and dimorphic anaemia, the RBC-histograms can be used for analysis of anaemias where the prevalence rates are high. Peripheral smear evaluation is still not being replaced by any parameter for the analysis of anaemias. Both the automation parameters and smear in conjunction help to achieve an accurate analysis of anaemias.

The present study was done on a rural population where anaemia prevalence is quite high and the population needs a test which is rapid yet accurate both for screening and confirmation. We analysed the role of $\mathrm{MCV}$ and RBC-histogram in the analysis of anaemia for their sensitivity, specificity and predictive values.

The present study is of the opinion that Peripheral smear examination cannot be replaced by Mean Corpuscular Volume estimation and RBC-histogram can be used with near complete accuracy in cases of dimorphic anaemia.

The present study is also of the opinion that rapid staining methods of the Peripheral smear can be very useful in decreasing the turn-around time of the reports instead of relying completely on the automated parameters obtained from the cell counter.
Findings: Nil; Conflict of Interest: None initiated Permission from IRB: Yes

\section{References}

1. Chaudhary SM, Dhage VR. A study of Anemia among adolescent females in the urban area of Nagpur. Indian J Community Med. 2008 Oct;33(4):243-5. doi: 10.4103/0970-0218.43230.DOI:10.4103/0970-0218. 43230

2. UNICEF. ADIÓS ANEMIA: A Local Strategy for the Reduction of Childhood Anemia in Peri-Urban Areas; UNICEF: Lima, Peru, 2012.

3. Bentley ME, Griffiths PL. The burden of anemia among women in India. Eur J Clin Nutr. 2003 Jan; 57 (1):52-60.DOI:10.1038/sj.ejcn.160 1504

4. Fossat C, David M, Harle JR, et al. New parameters in erythrocyte counting. Value of histograms. Archives of pathology \& laboratory medicine 111:12 1987 Dec: 1150-4.

5. Shrilekha Sairam, Suhasini Domalapalli, Sundaram Muthu, Jayanthi Swaminathan, Vivek A. Ramesh, Lalitha Sekhar, Palak Pandeya, and Udhaya Balasubramaniam. Hematological and Biochemical Parameters in Apparently Healthy Indian Population: Defining Reference Intervals. Indian J Clin Biochem. 2014 Jul; 29 (3): 290-297.

6. Thomas ETA, Bhagya S, Majeed A. Clinical Utility of Blood Cell Histogram Interpretation. J Clin Diagn Res. 2017 Sep; 11 (9): OE01-OE04. doi: 10.7860/ JCDR/2017/28508.10620.Epub2017 Sep 1.

7. International Institute for Population Sciences (IIPS) and Macro International. 2007. National Family Health Survey-3(NFHS-3),2005-06.India:Vol.1.Mumbai: IIPS.

8. Kawaljit Kaur. Anaemia 'a silent killer' among women in India: Present scenario. European Journal of Zoological Research, 2014, 3 (1):32-36

9. Matthew Little, Chloe Zivot, Sally Humphries, Warren Dodd, Kirit Patel, and Cate Dewey. Burden and Determinants of Anemia in a Rural Population in South India: A Cross-Sectional Study. Anemia Volume 2018, Article ID 7123976, 9 pages. https://doi.org/10.1155/ 2018/7123976

10. Pasricha SR. Anemia: a comprehensive global estimate. Blood. 2014 Jan 30;123(5):611-2.doi: 10.1182 / blood-2013-12-543405. 
11. Ezzati M, Lopez AD, Rodgers AA, Murray CJL. Comparative quantification of health risks: global and regional burelen of diseases attributable to selected major risk factor. Geneva, Switzerland: WHO,2004.

12. Sandhya I, Muhasin T. P. Study of Rbc Histogram in Various Anemias. Journal of Evolution of Medical and Dental Sciences. 2014; Vol.3, Issue 74, December 29; Page:15521 15534, DOI: 10.14260 / jemds / 2014/4095.

13. Byna Syam Sundara Rao, Vissa Shanthi, Nandam Mohan Rao, Bhavana Grandhi, Vijayalakshmi Muramreddy, Sirasala Praveena. RBC Histogram as Supplementary Diagnostic Tool with Peripheral Smear Examination in Evaluating Anaemia. Annals of Pathology and Laboratory Medicine, Vol. 4, Issue 6, November-December 2017.
14. Ashutosh Kumar, Rashmi Kushwaha, Chani Gupta, U. S. Singh. An analytical study on peripheral blood smears in anemia and correlation with cell counter generated red cell parameters. Journal of Applied Hematology. Vol. 4 Issue 4 October-December 2013.

15. Shruti Singla, Sanjay Bedi, Kusum Joshi. Comparative study of anemia cases based on peripheral blood smears MedPulse - International Medical Journal. January 2017; 4(1): 44-4.

16. Aseem Jain, Arvind Bhake, Malvika Tripathi. CoRelative Study on Peripheral Blood Smears in Anemia with Automated Cell Counter Generated Red Cell Parameters. IOSR Journal of Dental and Medical Sciences. Volume 17, Issue 3 Ver.16 March. (2018), PP 80-83.

\section{How to cite this article?}

O. Sirisha, M.N.P. Charan Paul, P. Yujwal Raj. Evaluation of role of mean corpuscular volume and RBC-histogram in analysing anaemias as a rapid method in comparison to peripheral smear evaluation. Trop J Path Micro 2019;5 (2): 76-82.doi:10.17511/jopm.2019.i02.05. 